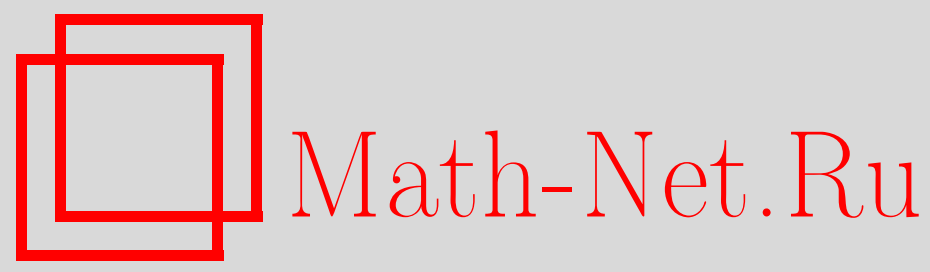

А. В. Устинов, Дискретный аналог формулы суммирования Эйлера, Матем. заметки, 2002, том 71, выпуск 6, 931-936

DOI: https://doi.org/10.4213/mzm397

Использование Общероссийского математического портала Math-Net.Ru подразумевает, что вы прочитали и согласны с пользовательским соглашением http://www. mathnet.ru/rus/agreement

Параметры загрузки:

IP: 54.198 .64 .247

26 апреля 2023 г., 13:22:04

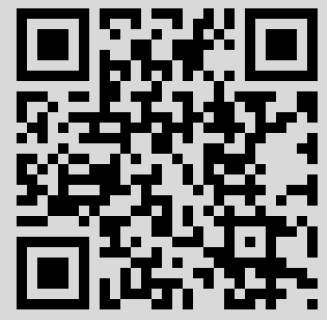




\section{ДИСКРЕТНЫЙ АНАЛОГ ФОРМУЛЫ СУММИРОВАНИЯ ЭЙЛЕРА}

\section{А. В. Устинов}

В работе доказывается дискретный аналог формулы суммирования Эйлера. Отличие от классического варианта формулы Эйлера заключается в том, что производные заменяются на конечные разности, интегралы - на конечные суммы. Вместо чисел и полиномов Бернулли в формуле появляются специальные числа $P_{n}$ и специальные полиномы $P_{n}(x)$, введенные Коробовым в 1996 году.

Библиографоял: 3 названия.

1. Введение. В работе [1] Коробовым были введены специальные числа $P_{n}$ и специальные полиномы $P_{n}(x)$, которые можно назвать дискретными аналогами чисел Бернулли $B_{n}$ и полиномов Бернулли $B_{n}(x)$. Аналогия заключается в том, что свойства полиномов $B_{n}(x)$ превращаются в свойства полиномов $P_{n}(x)$ при замене непрерьвных объектов на дискретные: производных на конечные разности, интегралов на конечные суммы, рядов Фурье на конечные ряды Фурье.

Одним из важных приложений полиномов Бернулли является формула суммирования Эйлера, которая устанавливает связь между суммой значений достаточно гладкой функции $f(x)$ в точках равномерной сетки

$$
\sum_{k=a}^{b-1} f(k)
$$

и определенньм интегралом от этой функции по соответствующему отрезку:

$$
\sum_{k=a}^{b-1} f(k)=\int_{a}^{b} f(x) d x+\left.\sum_{\nu=1}^{n} \frac{B_{\nu}}{\nu !} f^{(\nu-1)}(x)\right|_{a} ^{b}-R_{n}[f]
$$

где

$$
R_{n}[f]=\int_{a}^{b} \frac{B_{n}(1-\{x\})}{n !} f^{(n)}(x) d x=(-1)^{n} \int_{a}^{b} \frac{B_{n}(\{x\})}{n !} f^{(n)}(x) d x .
$$

Другим приложением полиномов Бернулли является формула для представления произвольной функции $f(x)$ на отрезке $[0 ; 1]$ :

$$
f(x)=\int_{0}^{1} f(y) d y+\sum_{k=1}^{n} \frac{B_{k}(x)}{k !}\left(\left.f^{(k-1)}(y)\right|_{0} ^{1}\right)-R_{n},
$$

Работа выполнена при частичной поддержке Российского фонда фундаментальных исследований, грант № 01-01-00738. 
где

$$
R_{n}=\int_{0}^{1} \frac{B_{n}(\{x-y\})}{n !} f^{(n)}(y) d y=(-1)^{n} \int_{0}^{1} \frac{B_{n}(1-\{x-y\})}{n !} f^{(n)}(y) d y
$$

(см., например, [2]).

При использовании формул (2) и (3) в прикладных задачах возникает неудобство, связанное с необходимостью знать производные функции $f(x)$.

В настоящей работе доказьваются дискретные аналоги формул (2) и (3) (теоремы 2 и 1). В дискретном аналоге формулы Эйлера сумма (1) приближается не интегралом, а суммой значений той же функции в узлах более густой равномерной сетки:

$$
\frac{1}{p} \sum_{y=a p}^{b p-1} f\left(\frac{y}{p}\right)
$$

В задаче о нахождении суммы (1) такая формула может оказаться более удобной, если сумма (4) подсчитьвается точнее, чем интеграл

$$
\int_{a}^{b} f(x) d x
$$

С другой стороны, дискретньй аналог формулы суммирования Эйлера представляется более удобным для использования в задачах приближенного анализа, так как эта формула связьвает сумму (4), близкую к интегралу (5), с более короткой суммой (1). Причем в отличие от обычной формулы суммирования Эйлера для применения ее дискретного аналога не требуется знать значения производных функции, а нужны лишь значения самой функции в конечном наборе точек.

2. Дискретный аналог формулы суммирования Эйлера. Пусть $n \geqslant 0, p \geqslant 2-$ целые числа. При любом действительном $x$ числа $P_{n}$ и полиномы $P_{n}(x)$ определяются равенствами

$$
\begin{gathered}
P_{0}=1, \quad C_{p}^{1} P_{n}+\cdots+C_{p}^{n+1} P_{0}=0 \quad(n \geqslant 1) \\
P_{0}(x)=1, \quad P_{n}(x)=P_{0} C_{x}^{n}+\cdots+P_{n-1} C_{x}^{1}+P_{n} \quad(n \geqslant 1)
\end{gathered}
$$

Пользуясь определением, нетрудно вычислить первые специальные числа и специальные полиномы

$$
\begin{gathered}
P_{1}=-\frac{p-1}{2}, \quad P_{2}=\frac{p^{2}-1}{12}, \quad P_{3}=-\frac{p^{2}-1}{24} \\
P_{1}(x)=x-\frac{p-1}{2}, \quad P_{2}(x)=\frac{1}{2} x^{2}-\frac{p}{2} x+\frac{p^{2}-1}{12} \\
P_{3}(x)=\frac{1}{6} x^{3}-\frac{p+1}{4} x^{2}+\frac{p^{2}+3 p}{12} x-\frac{p^{2}-1}{24} .
\end{gathered}
$$

Определим действие оператора конечной разности $\Delta$ при помощи равенств

$$
\Delta^{0} f\left(\frac{x}{p}\right)=f\left(\frac{x}{p}\right), \quad \Delta^{1} f\left(\frac{x}{p}\right)=f\left(\frac{x+1}{p}\right)-f\left(\frac{x}{p}\right) .
$$


Конечную разность порядка $n \geqslant 1$ определим с помощью рекуррентного соотношения

$$
\Delta^{n} f\left(\frac{x}{p}\right)=\Delta^{1}\left(\Delta^{n-1} f\left(\frac{x}{p}\right)\right) .
$$

Отметим два свойства специальных полиномов, доказанные в работе [1]:

$1^{\circ} . P_{n}(0)=P_{n}(n \geqslant 0)$;

$2^{\circ}$. при целом $x \in[0 ; p+n-2]$

$$
P_{n}(x)=-\sum_{m=1}^{p-1} \frac{e^{2 \pi i m / p}}{\left(e^{2 \pi i m / p}-1\right)^{n}} e^{2 \pi i m x / p} \quad(n \geqslant 0) .
$$

Докажем сначала дискретный аналог формулы (3).

Теорема 1. Пусть $n, k$ - челые числа, $n \geqslant 1$, и функция $f(z / p)$ определена во всех точках промежутка $k \leqslant z / p<k+1+n / p$. Тогда для всех челых $x$ из интервала $0 \leqslant x \leqslant p$ справедливо равенство

$$
f\left(k+\frac{x}{p}\right)=\frac{1}{p} \sum_{y=0}^{p-1} f\left(k+\frac{y}{p}\right)+\left.\frac{1}{p} \sum_{\nu=1}^{n} P_{\nu}(x) \Delta^{\nu-1} f\left(k+\frac{y}{p}\right)\right|_{y=0} ^{p}-R_{n}
$$

әде остаток $R_{n}$ может бъть записан двумя способами:

$$
\begin{aligned}
& R_{n}=\frac{1}{p} \sum_{y=0}^{p-1} \Delta^{n} f\left(k+\frac{y}{p}\right) P_{n}\left(p\left\{\frac{p+x-y-1}{p}\right\}\right), \\
& R_{n}=\frac{(-1)^{n}}{p} \sum_{y=0}^{p-1} \Delta^{n} f\left(k+\frac{y}{p}\right) P_{n}\left(p\left\{\frac{y-x-n-1}{p}\right\}\right) .
\end{aligned}
$$

ДокаЗАТЕЛЬСтво. Докажем сначала формулы (6) с остаточным членом (7). При $n=1$ необходимо проверить равенство

$$
f\left(k+\frac{x}{p}\right)=\frac{1}{p} \sum_{y=0}^{p-1} f\left(k+\frac{y}{p}\right)+\frac{1}{p}\left(x-\frac{p-1}{2}\right)[f(k+1)-f(k)]-R_{1} .
$$

Для этого преобразуем остаток $R_{1}$ :

$$
\begin{aligned}
R_{1}= & \frac{1}{p} \sum_{y=0}^{p-1} \Delta f\left(k+\frac{y}{p}\right) P_{1}\left(p\left\{\frac{p+x-y-1}{p}\right\}\right) \\
= & \frac{1}{p} \sum_{y=0}^{x-1} \Delta f\left(k+\frac{y}{p}\right)\left(x-y-1-\frac{p-1}{2}\right) \\
& +\frac{1}{p} \sum_{y=x}^{p-1} \Delta f\left(k+\frac{y}{p}\right)\left(p+x-y-1-\frac{p-1}{2}\right) \\
= & f(k+1)-f\left(k+\frac{x}{p}\right)+\frac{1}{p} \sum_{y=0}^{x-1} \Delta f\left(k+\frac{y}{p}\right)\left(x-y-1-\frac{p-1}{2}\right) .
\end{aligned}
$$


К последней сумме применим преобразование Абеля

$$
\sum_{y=0}^{p-1} F(y) \varphi(y)=F(p) \sum_{y=0}^{p-1} \varphi(y)-\sum_{y=0}^{p-1}(F(y+1)-F(y)) \sum_{z=0}^{y} \varphi(z),
$$

положив $\varphi(y)=\Delta f(k+y / p)$ и $F(y)=x-y-1-(p-1) / 2$ :

$$
\begin{aligned}
R_{1}+f\left(k+\frac{x}{p}\right)= & f(k+1)+\frac{1}{p}\left(x-p-1-\frac{p-1}{2}\right)[f(k+1)-f(k)] \\
& +\frac{1}{p} \sum_{y=0}^{p-1}\left[f\left(k+\frac{y+1}{p}\right)-f(k)\right] \\
= & \frac{1}{p} \sum_{y=0}^{p-1} f\left(k+\frac{y}{p}\right)+[f(k+1)-f(k)]\left(x-\frac{p-1}{2}\right) .
\end{aligned}
$$

Тем самым, равенство (9) доказано.

Далее применим индукцию. Предположим, что равенства (6), (7) справедливы для некоторого $n \geqslant 1$.

Из свойства $2^{\circ}$ следует, что для всех целых $x$ выполняется равенство

$$
P_{n}\left(p\left\{\frac{x}{p}\right\}\right)=-\sum_{m=1}^{p-1} \frac{e^{2 \pi i m / p}}{\left(e^{2 \pi i m / p}-1\right)^{n}} e^{2 \pi i m x / p} \quad(n \geqslant 0) .
$$

Суммируя это равенство, приходим к соотношениям

$$
\begin{gathered}
\sum_{y=0}^{p-1} P_{n}\left(p\left\{\frac{p+x-y-1}{p}\right\}\right)=0 \\
\sum_{z=0}^{y} P_{n}\left(p\left\{\frac{x+x-z-1}{p}\right\}\right)=P_{n+1}\left(p\left\{\frac{x}{p}\right\}\right)-P_{n+1}\left(p\left\{\frac{p+x-y-1}{p}\right\}\right) .
\end{gathered}
$$

Вновь применим преобразование Абеля (10) к остатку $R_{n}$, выбирая

$$
F(y)=\Delta^{n} f\left(k+\frac{y}{p}\right) \quad \text { и } \quad \varphi(y)=P_{n}\left(p\left\{\frac{p+x-y-1}{p}\right\}\right) .
$$

С учетом формул (12) и (13) приходим к нужному равенству:

$$
R_{n}=-\left.\frac{1}{p} P_{n+1}(x) \Delta^{n} f\left(k+\frac{y}{p}\right)\right|_{0} ^{p}+R_{n+1},
$$

что доказьвает формулу (6) с остаточным членом в первой форме (7).

Для доказательства второго представления остатка (8) достаточно воспользоваться равенством

$$
P_{n}\left(p\left\{\frac{p-1-x}{p}\right\}\right)=(-1)^{n} P_{n}\left(p\left\{\frac{x+n-1}{p}\right\}\right) \quad(n \geqslant 0),
$$

которое является прямым следствием формулы (11). Теорема доказана.

Докажем теперь дискретный аналог формулы суммирования Эйлера (2). 
ТЕОРема 2. Пусть $b>$ a $и$ и $n \geqslant 1$ - иелье числа и функиия $f(z / p)$ определена во всех точках промежутка $a \leqslant z / p<b+n / p$. Тогда справедливо равенство

$$
\sum_{k=a}^{b-1} f(k)=\frac{1}{p} \sum_{y=a p}^{b p-1} f\left(\frac{y}{p}\right)+\left.\frac{1}{p} \sum_{\nu=1}^{n} P_{\nu} \Delta^{\nu-1} f\left(\frac{y}{p}\right)\right|_{a p} ^{b p}-R_{n}[f],
$$

где остаток $R_{n}[f]$ может быть записан двумя способами:

$$
\begin{aligned}
& R_{n}[f]=\frac{1}{p} \sum_{y=a p}^{b p-1} \Delta^{n} f\left(\frac{y}{p}\right) P_{n}\left(p\left\{\frac{p-y-1}{p}\right\}\right), \\
& R_{n}[f]=\frac{(-1)^{n}}{p} \sum_{y=a p}^{b p-1} \Delta^{n} f\left(\frac{y}{p}\right) P_{n}\left(p\left\{\frac{y+n-1}{p}\right\}\right) .
\end{aligned}
$$

ДокАЗАТЕЛЬСТво. Если в равенстве (6) положить $x=0$ и просуммировать его по $k$ от $a$ до $b-1$, то получится формула (15) с остатком $R_{n}[f]$ в форме (16). Вторая форма записи остатка (17), как и в теореме 1 , является следствием формулы (14). Теорема доказана.

ЗАмЕчАниЕ. Сушествуют различные оценки остаточного члена в формуле суммирования Эйлера (2), зависящие от поведения производных функции $f(x)$ (см. [3]). Эти оценки основываются на геометрических свойствах многочленов Бернулли. Например, всегда справедливо неравенство

$$
\left|R_{2 n-1}\right| \leqslant \frac{(b-a)\left|B_{2 n}\right|}{(2 n) !} \max _{\xi \in[a ; b]}\left|f^{(2 n)}(\xi)\right| .
$$

По аналогии можно доказать, что для остаточного члена формулы (15) выполняется оценка

$$
\left|R_{2 n-1}[f]\right| \leqslant 2(b-a) p^{2 n}\left(\frac{\left|B_{2 n}\right|}{(2 n) !}+\frac{1}{p^{2}}\right) \max _{y \in[a p ; b p-1]}\left|\Delta^{2 n} f\left(\frac{y}{p}\right)\right| .
$$

Автор планирует посвятить отдельную статью изучению геометрических свойств специальных полиномов и различным оценкам остаточного члена формулы суммирования (15).

\section{3. Символический вывод дискретного аналога формулы суммирования} Эйлера. Известно, что формула Эйлера допускает нестрогий символический вывод, основанньй на формальной связи между опрераторами сдвига, дифференцирования и оператором конечной разности (см., например, [3]). Ее дискретный аналог также может быть получен при помоши формальных рассуждений. Приведенные ниже выкладки, естественно, нельзя считать доказательством. Они являются лишь аргументом в пользу существования такой формулы. Для символического вывода формулы воспользуемся производящей функцией последовательности специальных чисел. Как отмечалось в работе [1], эта функция имеет вид

$$
\sum_{n=0}^{\infty} P_{n} t^{n}=\frac{p t}{(t+1)^{p}-1}
$$


Будем обозначать через $E$ оператор сдвига, действие которого определяется равенством

$$
E f\left(\frac{y}{p}\right)=f\left(\frac{y+1}{p}\right)
$$

Пусть $Q=b-a$. Левая часть формулы (15) может быть записана в виде

$$
\sum_{k=a}^{b-1} f(k)=\left(1+E^{p}+E^{2 p}+\cdots+E^{(Q-1) p}\right) f\left(\frac{a p}{p}\right)=\frac{E^{Q p}-1}{E^{p}-1} f\left(\frac{a p}{p}\right) .
$$

Сумма значений функции в рациональных точках со знаменателем $p$ примет вид

$$
\frac{1}{p} \sum_{y=a p}^{b p-1} f\left(\frac{y}{p}\right)=\frac{1}{p}\left(1+E+E^{2}+\cdots+E^{Q p-1}\right) f\left(\frac{a p}{j}\right)=\frac{1}{p} \cdot \frac{E^{Q p}-1}{E-1} f\left(\frac{a p}{p}\right) .
$$

Если воспользоваться операторным равенством $E-1=\Delta$, то разность операторов, которые применяются в первом и во втором случае, будет иметь следующее символическое представление:

$$
\frac{E^{Q p}-1}{p}\left(\frac{p}{E^{p}-1}-\frac{1}{E-1}\right)=\frac{E^{Q p}-1}{p \Delta}\left(\frac{p \Delta}{(\Delta+1)^{p}-1}-1\right) .
$$

В правой части оказалась производящая функция специальных чисел с аргументом $\Delta$. Раскладывая ее в ряд по степеням $\Delta$, приходим к равенству

$$
\frac{E^{Q p}-1}{p}\left(\frac{p}{E^{p}-1}-\frac{1}{E-1}\right)=\frac{E^{Q p}-1}{p} \sum_{\nu=1}^{\infty} P_{\nu} \Delta^{\nu-1}
$$

Применяя найденное операторное равенство к $f(a p / p)$, получаем дискретньй аналог формулы суммирования Эйлера без остаточного члена:

$$
\sum_{k=a}^{b-1} f(k)-\frac{1}{p} \sum_{y=a p}^{b p-1} f\left(\frac{y}{p}\right)=\left.\frac{1}{p} \sum_{\nu=1}^{\infty} P_{\nu} \Delta^{\nu-1} f\left(\frac{y}{p}\right)\right|_{a p} ^{b p}
$$

\section{СПИСОК ЦИТИРОВАННОЙ ЛИТЕРАТУРЫ}

[1] Коробов Н. М. Специальные полиномы и их приложения // Диофантовы приближения. Матем. записки. Т. 2. М.: МГУ, 1996. С. 77-89.

[2] Коробов Н. М. Теоретикочисловые методы в приближенном анализе. М.: Физматгиз, 1963.

[3] Гельфонд А. О. Исчисление конечных разностей. М.: Наука, 1967. 